\title{
Navigating an Articulated Vehicle and Reversing with a Trailer
}

\author{
Ulf Larsson, Caj Zell, Kalevi Hyyppä and Åke Wernersson \\ Computer Science and Electrical Engineering \\ University of Luleå, S-971 87 LULEÅ, SWEDEN \\ email: ulf@sm.luth.se fax: int 46-92072082
}

\begin{abstract}
This paper describes two related tests; to navigate an articulated lawn mover and to reverse a mobile robot with a trailer. In both cases the vehicle s are to follow a preprecified trajectory. The navigation principal is based on measured directions to several identical beacons, consisting of strips of reflective tapes. The angular sensor is a rotating laser for the illumination of the beacons and a highly sensitive electro-optical receiver for detecting the directions to the beacons. A Kalman filter is used to combine the measurements from the odometers with the detected angles to the known position of the beacons.

To measure the angle between the robot and the trailer the same laser was used. This was done by placing two reflective beacons on the trailer. The repeatability was within 2 centimetre at low speed. The navigation of these two different types of vehicles turns out to be, essentially, the same problem. The sensitivities are different. Emphasis is on robust state estimation.
\end{abstract}

Keywords: Navigation, AGV, Trailer, Articulated lawn mover, Laser Anglemeter, Identical Reflective Beacons, Position Estimation, Sensor Feedback.

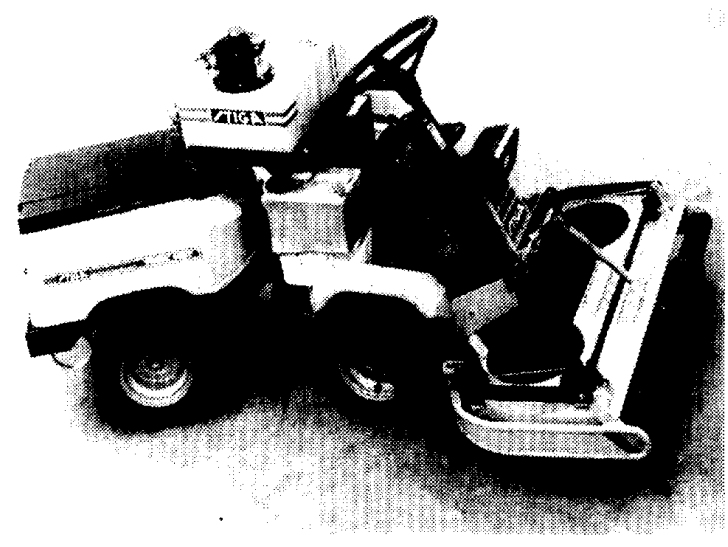

Figure 1 Photo of CALMAN. (Computerized Articulated Lawn Mower with Automatic Navigation). The rotating laser used to measure the directions to the reflecting tapes is seen on top of the lawn mover.

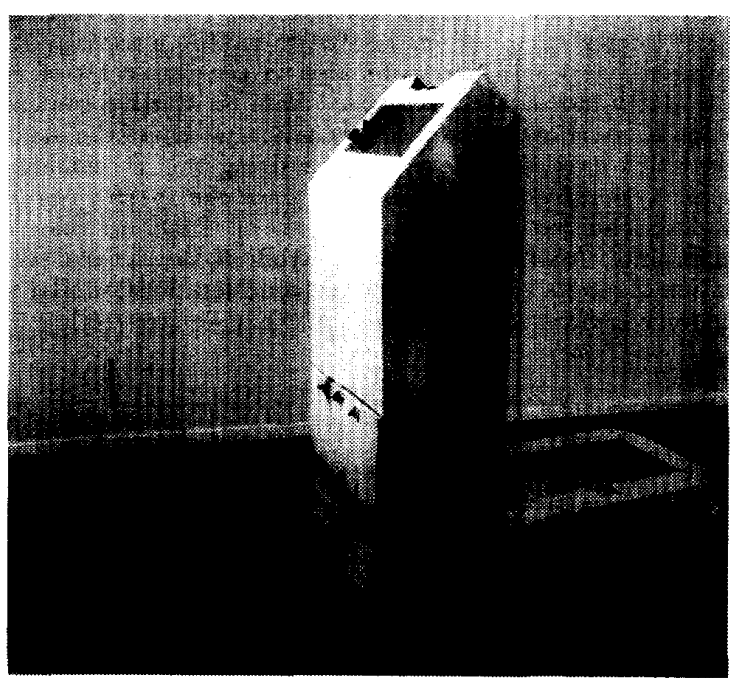

Figure 2 LTT (Lulea Turbo Turtle) with a trailer. The trailer is equipped with two reflective beacons. There is a column below the "tower" of LTT which makes it possible for the laser to detect the beacons on the trailer.

\section{Introduction}

A system for navigation using a rotating laser was proposed in [1]. A mobile robot is able to navigate accurately using identical retro reflecting tapes with known position stored in a map. The laser actively measures the directions to the tapes.

In two separate contributions, [4] and [5], to the Video Proceedings of IEEE Robotics and Automation the vehicles in figure 1 and figure 2 were presented. Video [4] was on navigating an autonomous lawn mover, CALMAN, while [5] was on reversing LTT with a trailer. In this paper the kinematics and navigation are described for both. It turns out that the two vehicles are, kinematically very similar.

Based on a position estimate the measurement from the laser is associated with one of the tapes in the map. An Extended Kalman Filter [EKF] is used to combine the 
information from odometers and directions given by the angular measuring laser, see also [2] and [3].

\subsection{The Angle Measuring Laser}

The continuously illuminating laser $(\leq 10 \mathrm{~mW})$ is of the same type as used in CD-players. The laser has internal correcting optics to produce a well collimated beam with a diameter of $5 \mathrm{~mm}$.

The optical path of the scanner goes through the vertical hollow axis of a rotating mirror enabling a 360 degree horizontal field of view of the scanner. The rotation angle of the rotating mirror is measured with an incremental encoder. The 1 speed of the scanner is 6 rotation/s and the error in measured angle is estimated to be less than 1 mrad.The main part of the receiver optics $i$ is a diamond-turned parabolic mirror. Details of the sensor are found in [2] and [6].

\subsection{CALMAN - The Autonomous Lawn Mower}

The platform used in this project is a standard articulated lawn mower. The purpose was to test the navigation algorithm outdoors. The main difference compared to earlier applications is that the mobile robot now is articulated also giving less accurate odometer measurements.

The lawn mover was equipped with a servo DC-motor for changing the articulation angle, actuators for changing the speed, for alternating between forward and reverse driving and for turning the cutter on and off. The internal sensors are incremental encoders for measuring both the articulation angle and the rotation of the non-driving front wheels (odometers).

\subsection{Reversing with a Trailer}

This project used the three wheeled mobile robot LTT (Luleå Turbo Turtle). The robot is controlled by changing the angle and speed of the front wheel. To measure the relative motion LTT is equipped with odometers on the rear wheels. For this project a small trailer was built. By putting two retro reflecting tapes on the posts on the trailer the rotating laser was also used to measure the angle between the robot and the trailer.

\section{Kinematics}

In order to control the trajectory of the robot we need a model of how the control variables affect the position of the vehicle. In this section we find a model that describes the motion of a vehicle consisting of multiple connected wheel axis.

\subsection{Kinematic Relations}

To derive a model we assume that the vehicle is moving on a flat surface with no slipping. The motion of each rigid body is then described by the speed $v_{n}$ and rotation $\omega_{n}$ of the midpoint of the axis. The motion of the body in a global coordinate system can be written as

$$
\left[\begin{array}{c}
\dot{x}_{n} \\
\dot{y}_{n} \\
\dot{\theta}_{n}
\end{array}\right]=\left[\begin{array}{c}
v_{n} \cos \theta_{n} \\
v_{n} \sin \theta_{n} \\
\omega_{n}
\end{array}\right]
$$

Thus, to describe the kinematics of the vehicles we need an expression for the rotation and speed of each wheel axis.

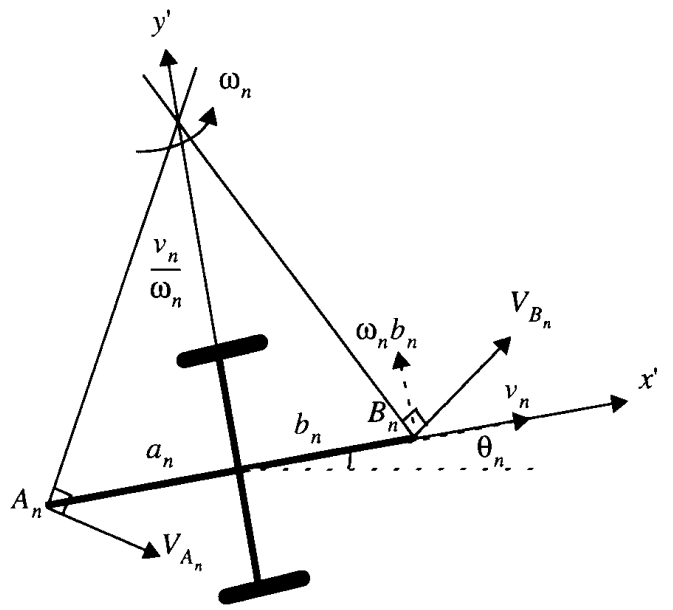

Figure 3 For each wheel axis the speed vectors for the points $A_{n}$ and $B_{n}$ can be expressed in a local coordinate base using $\omega_{n}$ and $v_{n}$. For example the $\mathrm{x}$ - and y-components for the speed vector at point $B_{n}$ are given by $v_{n}$ and $\omega_{n} b_{n}$ as indicated by the dashed arrows.

Due to the no slipping assumption $v_{n}$ must be perpendicular to the wheel axis. The speed vector on each rigid body at point $A_{n}$ and $B_{n}$ is given by (see figure 3)

$$
V_{A_{n}}=\left[\begin{array}{cc}
1 & 0 \\
0 & -a_{n}
\end{array}\right]\left[\begin{array}{l}
v_{n} \\
\omega_{n}
\end{array}\right], V_{B_{n}}=\left[\begin{array}{ll}
1 & 0 \\
0 & b_{n}
\end{array}\right]\left[\begin{array}{l}
v_{n} \\
\omega_{n}
\end{array}\right]
$$

where $V_{A_{n}}$ and $V_{B_{n}}$ are expressed in a coordinate base with y-axis along the wheel axis. If $A_{n}$ and $B_{n+1}$ are part of the same articulation joint then $V_{A_{n}}$ and $V_{B_{n+1}}$ describe the speed vector for the same point but in a different coordinate systems. The vector $V_{B_{n+1}}$ has the same length as $V_{A_{n}}$ but is rotated by $\varphi_{n}=\theta_{n}-\theta_{n+1}$.

$$
V_{B_{n+1}}=\left[\begin{array}{cc}
\cos \left(\varphi_{n}\right) & -\sin \left(\varphi_{n}\right) \\
\sin \left(\varphi_{n}\right) & \cos \left(\varphi_{n}\right)
\end{array}\right] V_{A_{n}}
$$

Combining eq. (2) and (3) gives us 


$$
\begin{gathered}
{\left[\begin{array}{c}
v_{n+1} \\
\omega_{n+1}
\end{array}\right]=T_{n}\left[\begin{array}{l}
v_{n} \\
\omega_{n}
\end{array}\right]} \\
T_{n}=\left[\begin{array}{cc}
1 & 0 \\
0 & b_{n+1}
\end{array}\right]^{-1}\left[\begin{array}{ll}
\cos \left(\varphi_{n}\right) & -\sin \left(\varphi_{n}\right) \\
\sin \left(\varphi_{n}\right) & \cos \left(\varphi_{n}\right)
\end{array}\right]\left[\begin{array}{cc}
1 & 0 \\
0 & -a_{n}
\end{array}\right]
\end{gathered}
$$

\subsection{The Articulated Vehicle}

In the case of an articulated vehicle (CALMAN) we let the state vector be the posture of the midpoint of the front axis

$$
\left[\begin{array}{lll}
x_{1} & y_{1} & \theta_{1}
\end{array}\right]^{T}
$$

The control signals are the speed of the front axis $v_{1}$ and the articulation angle $\varphi_{1}$. From eq. (4) we have

$$
\omega_{2}=\frac{1}{b_{2}}\left(v_{1} \sin \varphi_{1}-\omega_{1} a_{1} \cos \varphi_{1}\right)
$$

since $\omega_{2}=\omega_{1}-\dot{\varphi}_{1}$ this is rewritten as

$$
\omega_{1}=\frac{\sin \varphi_{1}}{b_{2}+a_{1} \cos \varphi_{1}} v_{1}+\frac{b_{2}}{b_{2}+a_{1} \cos \varphi_{1}} \dot{\varphi}_{1}
$$

In eq. (5) the first term on the right side is the change of heading when the robot moves with $\varphi_{1} \neq 0$. The second term describes how a change in articulation angle $\varphi_{1}$ affects the heading of the robot.

If we use the expression in eq. (5) for the rotation $\omega_{1}$ then it follows from eq. (1) that the kinematics of an articulated vehicle can be modelled as

$$
\left[\begin{array}{c}
\dot{x_{1}} \\
\dot{y_{1}} \\
\dot{\theta}_{1}
\end{array}\right]=\left[\begin{array}{c}
v_{1} \cos \theta_{1} \\
v_{1} \sin \theta_{1} \\
\frac{\sin \varphi_{1}}{b_{2}+a_{1} \cos \varphi_{1}} v_{1}+\frac{b_{2}}{b_{2}+a_{1} \cos \varphi_{1}} \dot{\varphi}_{1}
\end{array}\right]
$$

\subsection{Vehicle with a trailer}

When reversing with a trailer we need a model of how the midpoint of the trailer's wheel axis. The control signals are the speed $u$ and the direction $\alpha$ of the front wheel (see figure 4). The speed and rotation of the mobile robot is given by

$$
\left[\begin{array}{l}
v_{1} \\
\omega_{1}
\end{array}\right]=\left[\begin{array}{c}
u \cos \alpha \\
(u \sin \alpha) / b_{1}
\end{array}\right]
$$

Using eq. (4) we get

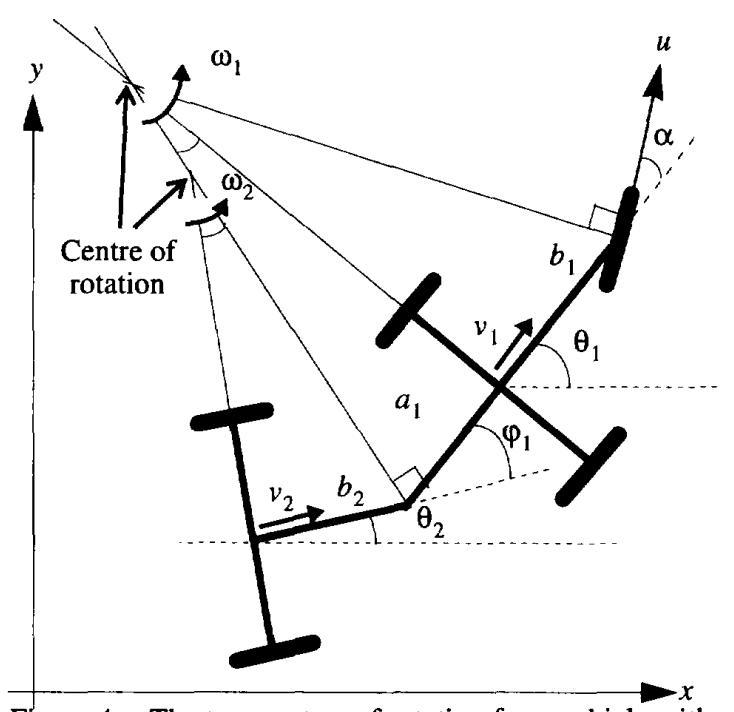

Figure 4 The two centres of rotation for a vehicle with a trailer. The similarity with an articulated vehicle are seen by removing the front wheel in this figure.

$$
\left[\begin{array}{l}
v_{2} \\
\omega_{2}
\end{array}\right]=\left[\begin{array}{l}
\left(\cos \varphi_{1} \cos \alpha+\frac{a_{1}}{b_{1}} \sin \varphi_{1} \sin \alpha\right) u \\
\frac{1}{b_{2}}\left(\sin \varphi_{1} \cos \alpha-\frac{a_{1}}{b_{1}} \cos \varphi_{1} \sin \alpha\right) u
\end{array}\right]
$$

To describe the motion of the mobile robot and the trailer we choose the state vector to be: The position of the midpoint of the trailers wheel axis $\left(x_{2}, y_{2}\right)$, the robots orientation $\theta_{1}$ and the angle between the robot and the trailer $\varphi_{1}$. Eq. (1) and the relation $\theta_{2}=\theta_{1}-\varphi_{1}$ give a continuous state space representation of the system

$$
\left[\begin{array}{c}
\dot{x}_{2} \\
\dot{y}_{2} \\
\dot{\theta_{1}} \\
\dot{\varphi_{1}}
\end{array}\right]=\left[\begin{array}{c}
v_{2} \cos \left(\theta_{1}-\varphi_{1}\right) \\
v_{2} \sin \left(\theta_{1}-\varphi_{1}\right) \\
\omega_{1} \\
\omega_{1}-\omega_{2}
\end{array}\right]
$$

where $v_{2}$ and $\omega_{2}$ are given by eq. (7).

\section{Estimating the Position}

To estimate the position of the robot the laser is used to measures the angles to the beacons. The motion of the vehicle between the time of the angle measurement is estimated by using odometers on the non-driven pair of wheels. We outline a model of how the odometers can be used to estimate the position and how to handle uncertainty in the estimate. The laser gives measurements 
between the times of update which have to be considered in the Extended Kalman Filter (EKF, see [8]).

\subsection{Model of Estimating the Position Using Odometers}

The motion of the robot is modelled by assuming that the robot moves along a circular segment during the sample interval. Let $l$ be the nominal distance between the wheels. Consider two points on the wheel axis, each at distance $l / 2$ from the midpoint of the wheel axis. For each sampling interval, denote the travelled distance for the points to the right resp. left of the midpoint $d_{r}(k)$ and $d_{l}(k)$. Define

$$
U(k)=\left[d_{r}(k) d_{l}(k)\right]^{T}
$$

The position $X(k)=[x(k) y(k) \theta(k)]^{T}$ of the midpoint of the wheel axis is updated as

$$
\begin{gathered}
X(k)=f(X(k-1), U(k))= \\
X(k-1)+\left[\begin{array}{c}
\frac{2 D(k) \sin (\Omega(k) / 2)}{\Omega(k)} \cos (\theta(k-1)+\Omega(k) / 2) \\
\frac{2 D(k) \sin (\Omega(k) / 2)}{\Omega(k)} \sin (\theta(k-1)+\Omega(k) / 2) \\
\Omega(k)
\end{array}\right]
\end{gathered}
$$

where $D(k)=\frac{d_{r}(k)+d_{l}(k)}{2}$ and $\Omega(k)=\frac{d_{r}(k)-d_{l}(k)}{l}$

Eq. (9) says that the midpoint of the wheel axis travels a distance $D(k)$ and rotates an angle $\Omega(k)$ between each sample time.

The travelled distance for each wheel is measured by the odometers at the sample time as $\tilde{d}_{r}(k)$ and $\tilde{d}_{l}(k)$. Define

$$
\tilde{U}(k)=\left[\tilde{d}_{r}(k) \tilde{d}_{l}(k)\right]^{T}
$$

The noise vector is introduced as $w(k)=U(k)-\tilde{U}(k)$ and approximated as Gaussian white noise. A preliminary model of the covariance to be used in the Kalman filter is

$$
\begin{aligned}
& Q(U(k))=\operatorname{cov}(w(k)) \approx \\
& {\left[\begin{array}{cc}
\left|\tilde{d}_{r}(k)\right| \sigma_{d}^{2}+\left|\frac{\tilde{d}_{r}(k)-\tilde{d}_{l}(k)}{l}\right| \sigma_{w}^{2} & 0 \\
0 & \left|\tilde{d}_{l}(k)\right| \sigma_{d}^{2}+\left|\frac{\tilde{d}_{r}(k)-\tilde{d}_{l}(k)}{l}\right| \sigma_{w}^{2}
\end{array}\right]}
\end{aligned}
$$

The second terms in diagonal elements are contribution to the error from the fact that the widths of the wheels are not zero, which will corrupt the odometer measurement when the robot rotates. For example, the wheels of CALMAN are thicker than the wheels of the mobile robot LTT. Thus by choosing larger values of $\sigma_{w}^{2}$ in the design of the EKF we somewhat considered the increase of the uncertainty in the motion estimate. To better understand the motion uncertainty further modelling and experiments are needed. Moreover, in the last covariance matrix the off diagonal terms are not modelled and put to zero.

Leaving the details the EKF is based on the approximation

$$
\begin{aligned}
& X(k)=f(X(k-1), \tilde{U}(k)+w(k)) \approx \\
& f(X(k-1), \tilde{U}(k))+G(X(k-1), \tilde{U}(k)) w(k)
\end{aligned}
$$

where $G(X(k-1), \tilde{U}(k))=\left.\frac{\partial}{\partial U} f(X(k-1), U)\right|_{U=\tilde{U}(k)}$

\subsection{Angle Measurements and the Association Problem}

At each sampling time we have a number of detected angles with time stamps from the laser scanner. Some of them correspond to known reflectors' positions $\left\{\left(x_{i}, y_{i}\right)\right\}$ but some of them may be false angles due to other reflecting objects (more about this problem is found in [7]). To make the navigation system work properly we only use detected angles which are alone in an acceptance window, see fig. 5 .

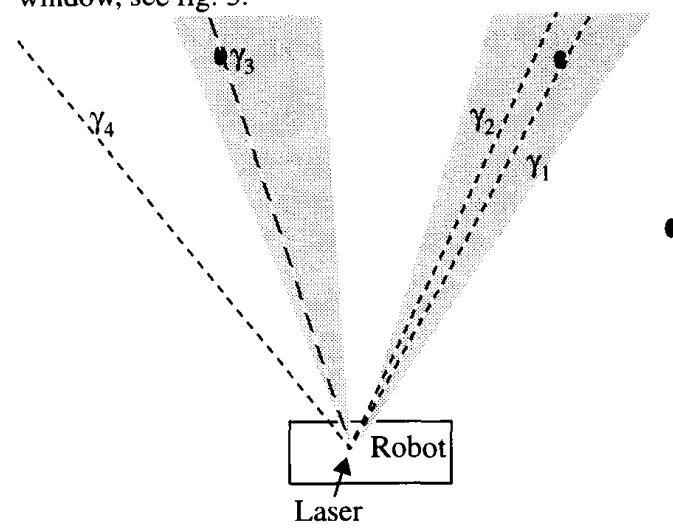

Figure 5 The rotating laser detect four angles. The dots in the figure are the known beacons. The gray areas are the acceptance windows. In this case only measurement $\gamma_{3}$ is accepted

For simplicity, the position of the scanning laser is assumed to be on the midpoint of the wheel axis. Let $\hat{X}(k)$ be the estimated position at the time when the measurements were taken. The formula

$$
\hat{\gamma}_{i}(k)=h(\hat{X}(k))=-\hat{\theta}+\operatorname{atan}\left(\frac{x_{i}-\hat{x}(k)}{y_{i}-\hat{y}(k)}\right)
$$

predicts the angles to all reflectors. 


\subsection{The Extended Kalman Filter}

When using the EKF we have to update the position in two steps. First we have to estimate the state vector when the measurement was taken. After we have improved the estimate using the angle measurement in the Kalman filter we estimate the current position.

Suppose that $\gamma$ is an accepted measured angle to a reflector and that the measurement is taken at time $t_{o b s}$. Let $t_{k-1}$ and $t_{k}$ be the time of two consecutive updates such that $t_{k-1}<t_{o b s}<t_{k}$. At $t_{k}$ we have information from the odometers describing the travelled distance for each wheel $\tilde{U}\left(t_{k}\right)$. Define

$$
U_{1}=\frac{t_{o b s}-t_{k-1}}{t_{k}-t_{k-1}} \tilde{U}\left(t_{k}\right), U_{2}=\frac{t_{k}-t_{o b s}}{t_{k}-t_{k-1}} \tilde{U}\left(t_{k}\right)
$$

We assume that the speed of the robot is constant during a sample period.The estimate of the position at time $t_{o b s}$ is given by using $U_{1}$ as the odometers value at the time $t_{o b s}$.

$$
\hat{X}\left(t_{o b s}\right)=f\left(\hat{X}\left(t_{k-1}\right), U_{1}\right)
$$

Eq. (12) is then used to find the associated beacon to $\gamma$ as described in the previous section. Assume that the covariance of the estimated position at $t_{k-1}$ is $\Sigma\left(t_{k-1} \mid t_{k-1}\right)$. Then the covariance for the position is updated as

$$
\begin{gathered}
\Sigma\left(t_{o b s} \mid t_{k-1}\right)=F\left(t_{k-1}\right) \Sigma\left(t_{k-1} \mid t_{k-1}\right) F\left(t_{k-1}\right)^{T} \\
+G\left(\hat{X}\left(t_{k-1}\right), U_{1}\right) Q\left(U_{1}\right) G\left(\hat{X}\left(t_{k-1}\right), U_{1}\right)^{T}
\end{gathered}
$$

where $F\left(t_{k-1}\right)$ is the Jacobian of $f\left(\hat{X}\left(t_{k-1}\right), U_{1}\right)$ with respect to $X$.The measurement $\gamma$ is now used to improve the estimate of $X$. Let $\hat{X}\left(t_{o b s}^{+}\right)$denote the filtered estimate of $X$ at time $t_{o b s}$

$$
\hat{X}\left(t_{o b s}^{+}\right)=\hat{X}\left(t_{o b s}\right)+K\left(t_{o b s}\right)\left(\gamma-h\left(\hat{X}\left(t_{o b s}\right)\right)\right)
$$

where the Kalman gain $K\left(t_{o b s}\right)$ is given by

$$
\begin{gathered}
K\left(t_{o b s}\right)=\Sigma\left(t_{o b s} \mid t_{k-1}\right) H^{T}\left(t_{o b s}\right) \\
\times\left(H\left(t_{o b s}\right) \Sigma\left(t_{o b s} \mid t_{k-1}\right) H^{T}\left(t_{o b s}\right)+S\right)^{-1}
\end{gathered}
$$

$H\left(t_{o b s}\right)$ is the Jacobian of $h\left(\hat{X}\left(t_{o b s}\right)\right)$ (eq. (11)) with respect to $X$ and $S=\operatorname{var}(\gamma)$. The covariance is updated as

$$
\Sigma\left(t_{o b s} \mid t_{o b s}\right)=\left(I-H\left(t_{o b s}\right) K\left(t_{o b s}\right)\right) \Sigma\left(t_{o b s} \mid t_{k-1}\right)
$$

By using $U_{2}$ we get an estimate of the position at time $t_{k}$ as

$$
\hat{X}\left(t_{k}\right)=f\left(\hat{X}\left(t_{o b s}^{+}\right), U_{2}\right)
$$

with the covariance

$$
\Sigma\left(t_{k} \mid t_{k}\right)=F\left(t_{o b s}\right) \Sigma\left(t_{o b s} \mid t_{o b s}\right) F\left(t_{o b s}\right)^{T}
$$

$$
+G\left(\hat{X}_{o b s}\left(t_{o b s}^{+}\right), U_{2}\right) Q\left(U_{2}\right) G\left(\hat{X}_{o b s}\left(t_{o b s}^{+}\right), U_{2}\right)^{T}
$$

\subsection{Estimating the Angle Between the Robot and the Trailer}

The laser scanner was also used to estimate the angle between the robot and the trailer (see figure 6).

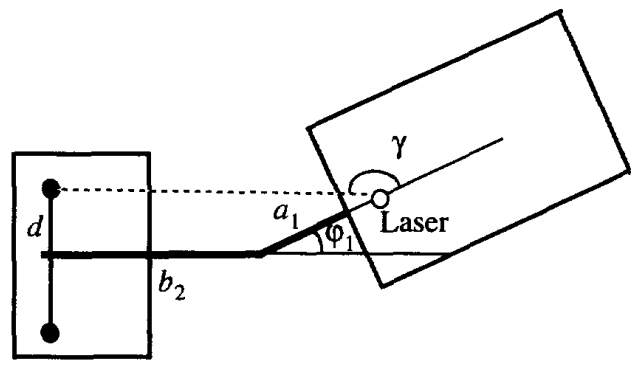

Figure 6 The angle $\varphi_{1}$ between the robot and the trailer is estimated by measuring the angle $\gamma$ to one of the two retro reflecting tapes on the trailer.

To use an EKF to estimate the angle $\varphi_{1}$ we need a discrete model of $\varphi_{1}$ during motion. Using eq. (8) and (4) gives

$$
\dot{\varphi}_{1}=\omega_{1}-\frac{1}{b_{2}}\left(v_{1} \sin \varphi_{1}-a_{1} \cos \varphi_{1}\right) \omega_{1}
$$

Assuming that $\omega_{1} v_{1}$ and $\varphi_{1}$ are constant during a sampling period we get

$$
\begin{gathered}
\varphi_{1}(k+1)=\varphi_{1}(k)+ \\
\left(1-\frac{D(k)}{b_{2}} v_{1} \sin \varphi_{1}(k)+\frac{a_{1}}{b_{2}} \cos \varphi_{1}(k)\right) \Omega(k)
\end{gathered}
$$

with $D(k)$ and $\Omega(k)$ as above. The predicted angle to one of the reflectors is given as

$$
\gamma=\operatorname{atan} \frac{d \cos \hat{\varphi}_{1}(k)+b_{2} \sin \hat{\varphi}_{1}(k)}{-a_{1}-b_{2} \cos \hat{\varphi}_{1}(k)+d \sin \hat{\varphi}_{1}(k)}+\pi
$$

Using eq (13) and (14) the odometers are combined with the angle measurements in an EKF as in the case of the position estimate.

\section{Control Laws}

The control of non-holonomic vehicles has been studied in several papers the last year, say, [11]. The problem of reversing with trailers has also been studied in, say, [12]. Since the empasis is on the state estimattion we used simple technique in designing the control laws

The desired trajectory of the robot is specified using consecutive straight line segments. The planning is manual programmed off-line.The problem of controlling 
the vehicle along the desired trajectory is then reduced to finding a control law that steers the robot along the $\mathrm{x}$-axis. In both applications the speed is assumed to be slowly varying. The control variables are the articulation angle for CALMAN and the direction of the steering wheel while reversing with the LTT. The speed controller is then designed such that the speed slows down when the errors are large.

\subsection{Steering CALMAN}

A well known steering algorithm is to have a real or fictitious steering wheel heading for a point on the $\mathrm{x}$-axis (see [9] and [10] for example). To use this technique we introduce a virtual as steering wheel in figure 7 . The virtual

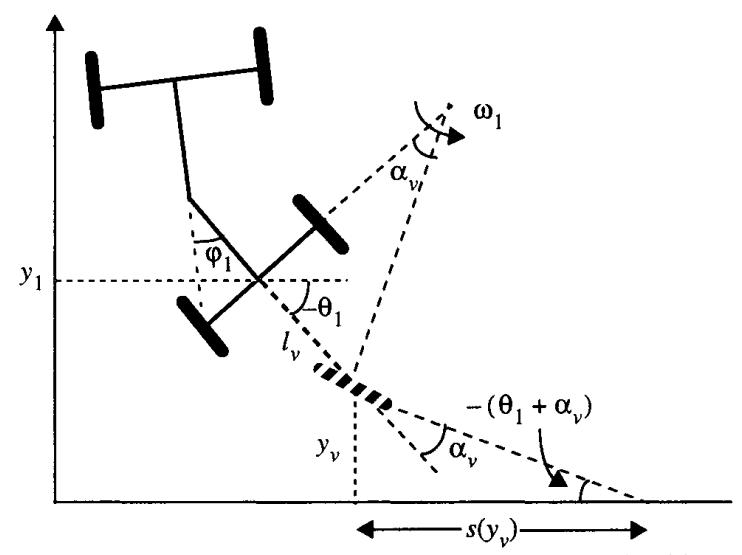

Figure 7 The control law. A virtual steering wheel is used to give the rotation of the front part. The virtual steering wheel is heading for a point on the $\mathrm{x}$ axis.

front wheel is heading towards a point at distance $s\left(y_{v}\right)$ on the $\mathrm{x}$ axis with $y_{v}=y+l_{v} \sin \theta$.

The position of the virtual steering wheel gives a desired direction for the wheel. Next the resulting rotation of the front part is calculated as

$$
\omega_{1}=v_{1} \frac{\tan \alpha_{v}}{l_{v}}=v_{1} \frac{\tan \left(\operatorname{atan}\left(-y_{v} / s\left(y_{v}\right)\right)-\theta\right)}{l_{v}}
$$

There are two design parameters that effect the performance. If $l_{v}$ is chosen small the robot steers towards the $\mathbf{x}-$ axis faster, but becomes less stable. $s\left(y_{v}\right)$ can also be chosen to give acceptable performance. More details are found in [9]. In the real system the input is given in discrete time.

To use the above control law we have to find an approximate solution to the heading dependence of the control variable. By assuming that $\varphi_{1}$ is constant during a sample period we get a change of heading shown as

$$
\begin{gathered}
\theta_{k+1}-\theta_{1}(k)=g\left(\varphi_{1}(k)\right)=\frac{T_{s} v \sin \varphi_{1}(k)}{b_{2}+a_{1} \cos \varphi_{1}(k)}+ \\
+\frac{b_{2}}{b_{2}+a_{1} \cos \varphi_{1}(k-1)}\left(\varphi_{1}(k)-\varphi_{1}(k-1)\right)
\end{gathered}
$$

The control signal $\varphi_{1}(k)$ is then calculated by solving the equation

$$
g\left(\varphi_{1}(k)\right)=\omega_{1} T_{s}
$$

By a first order Taylor expansion we get the following approximation

$$
\varphi_{1}(k) \approx \frac{\omega_{1} T_{s}-g\left(\varphi_{1}(k-1)\right)}{\left.\frac{d}{d \varphi_{1}} g\left(\varphi_{1}\right)\right|_{\varphi_{1}=\varphi_{1}(k-1)}}+\varphi_{1}(k-1)
$$

This control law is based on a continuous control law which assumes no dynamics. This means that the performance is better at slow speeds (which reduces the effects of the dynamics) and with short sampling time (decreases the discretization errors).

\subsection{Reversing using an LQ-controller}

Another approach was taken to reverse the trailer. The control algorithm was achieved by using linear feedback. The state vector components are the trailers distance to the $\mathrm{x}$-axis, the orientation of the robot and the angle between the robot and the trailer. Eq. (8) and (8) gives the continuous state space model

$$
\left[\begin{array}{c}
\dot{y_{2}} \\
\dot{\theta_{1}} \\
\dot{\varphi_{1}}
\end{array}\right]=\left[\begin{array}{c}
u\left(\cos \varphi_{1} \cos \alpha+\frac{a_{1}}{b_{1}} \sin \varphi_{1} \sin \alpha\right) \sin \left(\theta_{1}-\varphi_{1}\right) \\
(u \sin \alpha) / b_{1} \\
\frac{(u \sin \alpha)}{b_{1}}-\frac{u}{b_{2}}\left(\sin \varphi_{1} \cos \alpha-\frac{a_{1}}{b_{1}} \cos \varphi_{1} \sin \alpha\right)
\end{array}\right]
$$

A linear discrete state space model with time step $T_{s}$ is achieved by linearisation around the reference value $y_{2}=0, \theta_{1}=-\pi$ and $\varphi_{1}=0$.

$$
X(k+1)=\left[\begin{array}{ccc}
1 & -u T_{s} & u T_{s} \\
0 & 1 & 0 \\
0 & 0 & 1-\frac{u T_{s}}{b_{2}}
\end{array}\right] X(k)+\left[\begin{array}{c}
0 \\
\frac{u T_{s}}{b_{1}} \\
\frac{u T_{s}}{b_{1}}+\frac{u T_{s} a_{1}}{b_{1} b_{2}}
\end{array}\right] \alpha(k)
$$

With the state vector

$$
X(k)=\left[y_{1}(k) \theta_{1}(k)-\pi \varphi_{1}(k)\right]^{T}
$$

The control law is given by the linear state feedback 


$$
\alpha(k)=L X(k)
$$

where $L$ is the steady state solution determined by minimizing the quadratic criterion

$$
J=\sum_{k=0}^{\infty} X_{k} A X_{k}^{T}+\alpha_{k} B \alpha_{k}
$$

Using simulation the weights $A$ and $B$ were selected such that the feedback system has an acceptable performance.

\section{Experimental Tests}

Some of the tests are documented in the video proceedings [4] and [5].

\subsection{Navigating CALMAN}

Test runs were carried out on a parking lot outside the university. The positions of the reflecting beacons and the manually trajectory were loaded into the computer. At low speed $(0.3 \mathrm{~m} / \mathrm{s})$ the navigation error was less than $2 \mathrm{~cm}$. The motion of the robot was stable at a speed of $1 \mathrm{~m} / \mathrm{s}$. At the higher speed, $1.5 \mathrm{~m} / \mathrm{s}$, CALMAN reached instability. When driving forward and if a reversing is starting too fast the velocity of the vehicle and the sign of the velocity estimate have to be consistent. Otherwise, the lawn mower will be unstable and "protest by a temporary instability transient in the articulation joint". This "nervous behaviour" can be seen in the video. It should be mentioned that CALMAN was later improved to cut grass on a wet rainy field. Even when the driving wheels were spinning it could navigate.

\subsection{Reversing with a Trailer}

When a process disturbance is introduced, generated by slightly pushing the trailer sideways $5 \mathrm{~cm}$, the algorithm rapidly adapts to the situation and returns the trailer to the specified route. When a measurement disturbance is introduced, generated by concealing the trailer-beacons, the algorithm is able to control the trailer for some 5-10 seconds by using the model, but then "loses" the trailer. When the trailer is supposed to follow a rectangular path, the repeatability is within $\pm 2 \mathrm{~cm}$.

\section{Acknowledgements}

Several persons have been involved in these two projects, both students and members of staff. The navigation system in CALMAN was implemented in cooperation with AutoNavigator AB. This work was partially supported by NUTEK. A reviewer should also be mentioned.

\section{References}

[1] K. Hyyppä, "Optical navigation system using passive identical beacons", Intelligent Autonomous System, Amsterdam 1986 (also US patent 4811228, 1989).

[2] U. Wiklund, U. Andersson and K. Hyyppä, "AGV navigation by angle measurements", Proc. 6th int. Conf. Automated Guided Vehicle System, pp 199-212 1988.

[3] U. Wiklund, "Algorithms for navigation of autonomous guided vehicles based on measurements of directions to identical beacons", Licentiate Thesis 1988:13L, Luleå University of Technology 1988.

[4] U. Sandberg, P. Åhman, U. Larsson, K. Hyyppä, §. Wernersson, et al., "CALMAN- Computerized Articulated Lawn Mower with Automatic Navigation", Video Proc. IEEE Conf. Robotics and Automation, 1992

[5] C. Zell, et. al., "Reversing an AGV with a trailer using direction to passive beacons" Video Proc. IEEE Conf. Robotics and Automation, 1993

[6] K. Hyyppä," On a laser anglemeter for mobile robot navigation", Doctoral Thesis 1993:117D, Luleå University of Technology 1993

[7] $\AA$. Wernersson. U. Wiklund, U. Andersson and K. Hyyppä, "Vehicle navigation using image information: on association errors", Intelligent Autonomous System 2, pp 8148221989.

[8] B. D. O. Anderson, J. B. Moore, "Optimal Filtering". Prentice Hall, 1979

[9] U. Andersson, "Trajectory estimate and control of autonomous guided vehicles". Licentiate Thesis 1989:10L, Luleå University of Technology 1989.

[10] F. Weiczer,"On the Navigation of Wheeled Mobile Robots", Licentiate Thesis, Chalmers University of Technology, Göteborg 1992.

[11] C.Samson and K.Ait-Abderrahim "Feedback Control of a Nonholonomic Wheeled Cart in Cartesian Space", Proc. IEEE Conf. Robotics and Automation, 1991

[12] D.Tilbury, J-P. Laumond, R.Murray, S. Sastry, G. Walsh "Steering Car-like System with Trailers Using Sinusoids" Proc. IEEE Conf. Robotics and Automation, 1992 